\title{
О состоянии и развитии системы мониторинга инженерных конструкций башни «Эволюция». Часть 2. Рекомендации по развитию
}

\author{
А.М.Белостоцкий, НИЦ СтаДи0, Москва \\ П.А.Акимов, НИУ МГСУ, Москва \\ Т.Б.Кайтуков, НИУ МГСУ, Москва \\ Н.0.Петряшев, НИЦ «Строительство», Москва \\ С.0.Петряшев, НИЦ «Строительство», Москва
}

Во второй части статьи ${ }^{1}$ представлены обоснованные рекомендации по дополнению системы мониторинга в части пространственно-координатного мониторинга с помощью ГЛОНACC/GPS-датчиков, а также контроля за фасадными конструкциями (контрольные параметры для светопрозрачных фасадных систем; оборудование для мониторинга фасадных систем). В завершении сформулированы рекомендации по разработке и интеграции адаптивных конечноэлементных моделей несущих и фасадных конструкций. Даны указания на разработки авторов, посвящённые оригинальной расчётноэкспериментальной методике динамического мониторинга несущих конструкций высотных зданий в части её общих положений и структурной схемы, параметризируемых конечноэлементных моделей, адаптации (калибровки) конечноэлементных моделей по данным инструментальных наблюдений, оценки несущей способности для фактического состояния и планирования измерений по результатам мониторинга.

Ключевые слова: математическое моделирование, численное моделирование, компьютерное моделирование, численные методы, метод конечных элементов, механическая безопасность, напряжённо-деформированное состояние, адаптивные конечноэлементные модели, система мониторинга инженерных конструкций, башня «Эволюция».

On the State and Development of Structural Health Monitoring System of the "Evolution" Tower. Part 2. Development Recommendations

\section{A.M.Belostotsky, SRC StaDy0, Moscow}

P.A.Akimov, NRU MGSU, Moscow

T.B.Kaytukov, NRU MGSU, Moscow

N.0.Petryashev, Research Center of Construction, Moscow

S.0.Petryashev, Research Center of Construction, Moscow

The paper presents the substantiated recommendations for the modification of the SHM system. Corresponding recommendations deal with spatial coordinate monitoring using GLONASS / GPS sensors and monitoring of facade structures (control parameters for translucent facade systems; equipment for monitoring facade

1 Первая часть статьи «0 состоянии и развитии системы мониторинга инженерных конструкций башни “Эволюция"» опубликована в № 1 журнала «Academia. Архитектура и строительство» за 2020 год. systems). Besides, recommendations for the development and integration of adaptive finite element models of load-bearing and facade structures are provided. The necessary links are given to the authors' papers devoted to developed computational and experimental methodology of structural health monitoring dealing with load-bearing structures of unique buildings in terms of formulation of original basic theoretical foundations of the advanced methodology of structural health monitoring, parameterized finite element models of buildings ("design" and "monitoring-oriented" models), adaptation (calibration) of finite element models in accordance with results of measurements, structural evaluation in a real situation of the building.

Keywords: mathematical modelling, numerical modelling, computer modelling, numerical methods, finite element method mechanical safety, stress-strain state, adaptive finite element models, structural health monitoring, "Evolution" tower.

\section{3. Рекомендации по дополнению системы мониторинга}

3.1. Рекомендации по дополнению СМИК

Основываясь на международном опыте мониторинга высотных зданий [6; 21; 23; 27], а также на соответствующей проектной документации, авторы предлагают следующие мероприятия: установка системы ГЛОНACC/GPS для измерения смещения здания; установка метеостанции для измерения скорости и направления ветра, температуры и др.; установка дополнительных акселерометров для определения первых собственных частот и форм исследуемого здания; проведение периодического мониторинга состояний железобетонных перекрытий.

Как известно [1], мониторинг состояния здания с использованием спутниковых технологий является оптимальным дополнением таких традиционных систем, как тензометры, уклономеры, наклономеры, традиционных геодезических наблюдений за осадками и кренами зданий. Данный метод наблюдений позволяет определять положение фиксированных точек строительных конструкций зданий (сооружений) в реальном времени, а также даёт возможность получения детального описания эксплуатационных характеристик сооружения в течение длительного периода времени. Преимущество такого мониторинга состоит в его непрерывном характере, в том числе в реальном времени, а также в наличии возможности оповещения заинтересованных лиц о достижении критиче- 
ских перемещений. Система высокоточных ГлоНACC/GPSприёмников включает комплект оборудования и программного обеспечения. Система предназначена для автоматического мониторинга за смещениями верхней части здания относительно неподвижного исходного пункта. В систему ГлоНАСС/ GPS должно быть включено минимум два приёмника, один из которых устанавливается на последнем этаже, второй, являясь неподвижной относительной точкой, - на уровне земли. В качестве датчика при мониторинге смещений верхней части здания было предложено использовать ГЛОНАCC/GPS-приёмник типа Leica GRX1200GG Pro (или аналог), антенна которого монтируется на верхней точке здания (рис. 1 a).

Точные координаты в такой ситуации определяются относительно исходного пункта, оборудованного ГЛОНАCC/GPSприёмником типа Leica GRX1200GG Pro (или аналог). Следует отметить, что спутниковые приёмники серии Leica GRX1200 специально разработаны для использования на базовых станциях и являются частью комплекта геодезических инструментов Leica System 1200. ГНСС-приёмники Leica GRX1200 представляют собой высокоточные спутниковые средства измерений, работающие с сигналами глобальных спутниковых систем позиционирования GPS и ГЛОНАСС. Приёмник Leica GMX 902GG специально разработан для применения в системах геодезического мониторинга. Реализована интеграция приёмников этого типа с программными продуктами Leica GNSS Spider, GeoMos и GNSS QC. Спутниковый навигационный приёмник Leica GMX902 определяет координаты с точностью до 3 мм + 0,5 ppm. 0бработка данных и выдача информации в таких ситуациях может и должна осуществляться в реальном времени с помощью соответствующих пакетов программ (типа Leica GPS Spider, GNSS QC) и программного обеспечения (П0) СМИК. Приёмники Leica GRX1200 позволяют получать некоррелированные спутниковые данные высокого качества практически в любых условиях, что делает их идеальными при создании базовых станций. Кроме того, приёмники GRX1200 с технологией SmartTrack+ разработаны с возможностью отслеживания ГНСС сигналов, таких как GPS L5 и Galileo в будущем, что даёт возможность развития системы. С помощью сети ГЛОНАCC/GPS приёмников возможно измерение динамических параметров колебания здания при ветровом воздействии в режиме реального времени, реализуется последующая постобработка результатов для получения

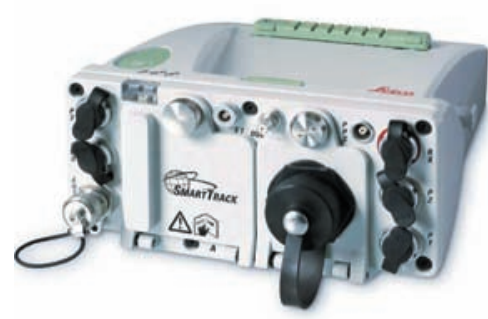

a)

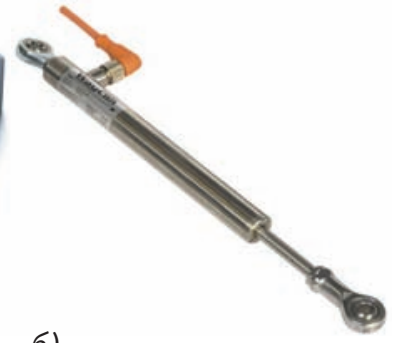

Рис. 1. Оборудование для мониторинга: a) средство измерения приращений координат Leica GRX1200GG Pro; б) индуктивный датчик линейных приращений (LVDT) WayCon данных с заданной точностью. Должно быть обеспечено вычисление изменения положения GLONASS/GPS-приёмника в контролируемой точке относительно исходных пунктов с точностью до 10 мм и с частотой 10...20 Гц. Приёмники ГЛОНАСС/ GPS устанавливаются жёстко с помощью стальных анкеров на несущие конструкции в зонах прямой видимости неба. Один приёмник устанавливается на кровле, один приёмник - на неподвижном пункте вне здания. Привязка реперных точек к осям здания «Эволюция» может быть осуществлена по утверждённому генплану застройки участка. С учётом известного проектного положения верха здания и базовой станции возможна поправка значений измерений перемещений верха здания на момент установки оборудования. Таким образом будет обеспечено измерение абсолютного отклонения верха здания от проектного положения. Система контроля состояния зданий с помощью ГлОНАCC/GPS-аппаратуры работает следующим образом. Координаты базовой точки (установленной на кровле) вследствие колебаний здания будут непрерывно изменяться, но - в некоторой известной области. При этом факт перемещений верха здания и его величина регистрируются автономно и независимо от того, какая причина их вызвала, - просадка или смещение грунта, разрушение элементов конструкции здания, внешнее воздействие, последствия пожара, террористический акт и т.д. Информация о смещении базовой точки передаётся в режиме реального времени через глобальную сеть «Интернет» или по другим сетям на удалённые терминалы.

Была предложена установка на кровле башни метеостанции (системы измерения скорости и направления ветра). Рассматриваемое здание является уникальным объектом с достаточно сложными, объёмно-планировочными и конструктивными решениями, не имевшими каких-либо аналогов. В СП 20.13330.2011², а также в других отечественных и зарубежных нормативных документах не приводились данные о распределении ветровой нагрузки по ограждающим конструкциям для сооружений подобного типа. Обычно в подобных случаях эти нагрузки традиционно определяются на основе данных модельных аэродинамических испытаний. Следует отметить, что результаты испытаний сооружений подобного типа показывают, что кроме их геометрической формы на величину и распределение расчётных ветровых нагрузок могут оказывать влияние несколько факторов, которые обычно не учитываются в практике проектирования. Здесь в первую очередь необходимо подчеркнуть, что на рассматриваемом участке расположены другие здания и сооружения, которые способствуют изменению структуры ветрового потока и, как следствие, изменению действующих ветровых нагрузок. Для данного объекта было проведено экспериментальное изучение распределения ветровых нагрузок по ограждающим поверхностям модели здания в специализированной аэродинамической трубе фирмы ВМТ (Лондон, Великобритания). На основании результатов экспе-

${ }^{2}$ СП 20.13330.2011 Нагрузки и воздействия. Актуализированная редакция СНиП 2.01.07-85*. - М. : ФГУП ЦПП, 2011. 
римента были выданы рекомендации по назначению ветровых нагрузок на ограждающие конструкции. В связи с тем, что актуальное состояние застройки территории ММДЦ «Москва-Сити» имеет ряд изменений в сравнении с проектом, результаты аэродинамических испытаний и соответствующие рекомендации не в полной мере соответствуют действующим на ограждающие конструкции ветровым нагрузкам. Установка метеостанции на кровле позволила бы уточнить ветровой режим вокруг здания. В частности, была предложена модель метеорологического комплекса МА-6-3 (или аналог), разработанного в НП0 «Тайфун». Данный наземный комплекс предназначен для автоматического измерения и вычисления основных метеорологических параметров, ввода дополнительно измеренных параметров, архивирования данных, формирования сообщений и передачи метеорологической информации в стандартные каналы связи в коде КН-01 с участием метеонаблюдателя.

Помимо установленных акселерометров в действующей системе СМИК была предложена установка дополнительных акселерометров для полного контроля собственных частот исследуемого здания. Согласно проекту, акселерометры должны были быть установлены на одиннадцати уровнях (каждые пять этажей), фактически же акселерометры установлены лишь на трёх уровнях (-3, 26 и 51 этажи). Согласно Приложению 3.2 МГСН 4.19-05³ приборы, измеряющие колебания конструкции, необходимо устанавливать через каждые пять этажей. В соответствии с указанными документами было рекомендовано установить дополнительные акселерометры на 0, 6, 11, 16, 21, 31, 36, 41 и 46 этажах. Анализ доступных способов определения не только собственных частот, но и форм колебаний показал, что метод стоячих волн (построенный на основе принципа восстановления когерентных составляющих волновых полей), разработанный коллективом Геофизической службы Сибирского отделения Российской академии наук (Новосибирск) под руководством доктора технических наук А.Ф. Еманова [2; 3; 5], позволяет определять не только низшие общесистемные, но и те собственные частоты и формы колебаний, которые идентифицируют локальные изменения состояния конструкций (включая разрушения), при выполнении также требований оперативности и экономической конкурентоспособности. Необходимая полная система наблюдений предусматривает одновременную регистрацию ускорений в опорной точке и некотором представительном наборе точек. Принимается модель линейной связи волновых полей в двух точках объекта. На основе винеровской фильтрации и свойства когерентности стоячих волн (колебаний) был разработан алгоритм расчёта фильтров, пересчитывающих колебания из опорной точки в другие точки системы наблюдений. В России уже накоплен позитивный опыт использования этого метода для определения собственных частот и форм колебаний плотин, мостов и зда-

${ }^{3}$ МГСН 4.19-2005. Временные нормы и правила проектирования многофункциональных высотных зданий и зданий-комплексов в городе Москве. - М., 2005. ний (большепролётных и, в меньшей степени, многоэтажных). Применительно к высотным зданиям-комплексам результаты исследований на реальном объекте приведены в [4].

Для оценки текущего состояния несущих конструкций перекрытий зданий, как известно, требуется осуществление непрерывного или периодического контроля. Для плит перекрытия, на которых при проведении строительных работ были выявлены зоны значительного трещинообразования, было предложено осуществление периодического мониторинга: визуальная (выявление дефектов) и инструментальная (измерение прогибов) оценка технического состояния перекрытий. Во время работ, приводящих к значительному увеличению нагрузки на перекрытия, необходим регулярный контроль их состояния - не реже одного раза в месяц. Альтернативным вариантом является проведение непрерывного мониторинга прогибов перекрытий.

При мониторинге состояния конструкции её фактическое состояние оценивается по глобальным [наблюдения за выбранными резонансными частотами (выводы об изменениях общей жёсткости); мониторинг изменений доминирующих форм колебаний] и локальным (измерение длины и ширины известных единичных трещин; наблюдение за деформациями в элементах конструкции, подверженных повышенной опасности образования трещин; деформации в местах с повышенной концентрацией напряжений; смещение конструктивных элементов; горизонтальное перемещение верха здания; осадка и крен фундамента) параметрам конструкции. Оценка несущей способности выполняется по действующим нормативным критериям на базе параметров НДС, определённых на текущей стадии мониторинга.

\section{2. Рекомендации по дополнению системы мониторинга фасадных конструкции}

В ходе мониторинга должен осуществляться контроль по следующим позициям: контроль НДС ограждающих конструкций эксплуатируемого здания; контроль геометрических параметров взаимного расположения отдельных компонентов фасадных систем; контроль климатических параметров в приграничных (с атмосферой) зонах наружных ограждений (показатели давления ветра, температура) (при необходимости). Необходимо провести сопоставление полученных параметров состояния контролируемых конструкций с нормируемыми параметрами, определёнными в проекте (или нормативными документами), а также оценку соответствия конструкций наружных ограждений зафиксированным климатическим воздействиям, в том числе в части проверки расчётных усилий в монтажных элементах. При мониторинге фасадных систем оцениваются следующие элементы и узлы: элементы крепления; поверхности несущих конструкций здания (колонны, ригели) под устройство навесных систем (геодезическая съёмка); крепёжные элементы на несущую способность; несущие элементы системы; узлы крепления несущих профилей; деформационные швы, узлы примыканий; стыки светопрозрачных элементов; выполнение узлов примыкания навесных светопрозрачных систем к другим системам, применённым на здании. 
Мониторинг фасадов позволяет своевременно выявить дефекты и выдать рекомендации для их устранения. При выявлении мест критических изменений НДС ограждающих конструкций производится обследования этих зон, выполняется оценка технического состояния конструкций, устанавливаются причины возникновения критического состояния и определяется необходимость проведения мероприятий по восстановлению, усилению или замене конструкций (а также вносятся изменения в программу мониторинга).

Была предложена установка по 12 датчиков ветрового давления на 17-ом 34-ом, 50-ом этажах (36 датчиков), причём на каждой стороне фасада здания на указанных этажах устанавливается по три датчика ветрового давления. Это связано с тем, что для выбранных этажей имеются результаты экспериментальных исследований в аэродинамической трубе. Таким образом, фактические значения ветрового давления могут сравниваться со значениями, учтёнными на стадии проекта. Было рекомендовано проводить мониторинг изменения деформационных швов фасадных элементов, в районе которых будут установлены датчики, по сравнению с соседними. Для измерения изменений деформационных швов была предложена установка индуктивных датчиков линейных перемещений (LVDT) WayCon (cм. рис. 1 б) или аналога - данное оборудование обладает высокой точностью измерений. Для проведения наблюдений были рекомендованы различные инструментальные системы (датчики ветрового давления; индуктивные датчики линейных перемещений и др.).

Необходимым является обследование направляющих и узлов их крепления (отклонения от проектного положения по вертикали и по горизонтали), а также контроль плотности соединений по критериям фирмы производителя навесной светопрозрачной системы.

\section{4. Рекомендации по разработке и интеграции адаптивных конечноэлементных моделей}

Основной недостаток системы датчиков и вывода информации с них заключается в том, что отсутствует полная картина распределения НДС по всей конструкции башни, так как количество датчиков и параметров состояния ограниченно.

В большинстве случаев для объекта мониторинга возможно выделить конечный набор независимых типовых нагрузок, возникающих в штатном режиме эксплуатации. На основе конечноэлементной модели необходимо провести моделирование отклика конструкции на эти нагрузки, рассчитать соответствующие НДС конструкции (деформации, напряжения и смещения в местах размещения датчиков).

«Мониторинговые» модели (или одна параметризуемая), как известно, имеют ряд специфических отличий от расчётных моделей, используемых для обоснования проектных решений: реализация не проектных, а фактических физико-механических свойств строительных материалов (бетона, арматуры и др.) и геометрии конструкции; учёт не нормативных, а фактических измеренных нагрузок и воздействий; включение в статическую и в особенности в динамическую работу при слабых «фоновых» воздействиях номинально ненесущих конструкций (перегородок, фасадных и др.); работа ряда узлов и соединений по схемам, отличным от принятых в проекте (например, упругая заделка вместо шарнира); адаптируемость (калибруемость, «обучаемость») модели по данным инструментального мониторинга, в том числе включение обнаруженных дефектов.

В целом, только система инструментального мониторинга, построенная на основе анализа результатов конечноэлементного моделирования в сопоставлении с данными измерений, позволит выполнить планирование мероприятий по подготовке и реагированию на изменения ответственных конструкций, сделать обоснованные выводы о фактическом состоянии и возможности дальнейшей безопасной эксплуатации здания ${ }^{4}[6-29]$.

0бщие положения и структурная схема такой методики описана в $[7 ; 9 ; 14 ; 25]$. В частности, в этих работах предложены параметризованные конечноэлементные модели зданий, алгоритм их адаптации (калибровки) по данным инструментальных наблюдений, методика измерения собственных частот и форм колебаний, подход к оценке несущей способности для фактического состояния объекта. «Стартовая» конечноэлементная модель объекта используется, как правило, для обоснования несущей способности актуального проектного варианта. Для каждой значимой стадии «жизненного цикла» здания (этапы строительства и эксплуатации) строится, модифицируется (актуализируется), верифицируется и адаптируется по текущим данным инструментальных наблюдений параметризуемая пространственная динамическая «мониторинговая» конечноэлементная модель. Основным адаптационным критерием здесь принимается соответствие расчётного и измеренного спектра собственных частот и форм колебаний во всём диапазоне частот, значимом как для оценки общесистемных изменений, так и идентификации-локализации возможных дефектов.

\section{Лuтература}

1. Белостоцкий, А.М. Научно-исследовательский центр СтаДи0. 25 лет на фронте численного моделирования / А.М. Белостоцкий, П.А. Акимов // International Journal for Computational Civil and Structural Engineering. - 2016. - Vol. 12, Is. 1. - P. 9-34.

2. Еманов, А.Ф. Восстановление когерентных составляющих волновых полей в сейсмике. Диссертация на соискание ученой степени доктора технических наук по специальности 25.00.10 - «Геофизика, геофизические методы поиска полезных ископаемых» / А.Ф. Еманов. - Новосибирск : Алтае-Саянский филиал Геофизической службы, Сибирское отделение РАН, 2004. - 279 с.

${ }^{4}$ ТР П-119-03-СМ-02-2010 Технический регламент по проведению научнотехнического сопровождения и мониторинга строительства большепролётных, высотных и других уникальных зданий и сооружений. 
3. Еманов, А.Ф. Применение метода стоячих волн для исследований сейсмоизолированных зданий / А.Ф. Еманов, А.А. Красников // Вопросы инженерной сейсмологии. - 2015. - № 4 (42). - С. 37-64.

4. Каличава Д.К. Адаптивные динамические конечноэлементные модели в основе мониторинга несущих конструкций высотных зданий. Диссертация на соискание учёной степени кандидата технических наук по специальности 05.13.18 «Математическое моделирование, численные методы и комплексы программ» / Д.К, Каличава. - М. : МГСУ, 2012. - 149 с.

5. Красников, А.А. Оценка полноты конечноэлементных моделей инженерных сооружений по экспериментальным данным метода стоячих волн / А.А. Красников, А.Ф. Еманов, А.А. Бах // Интерэкспо Гео-Сибирь. - 2017. - № 4 (2). - С. 179-184.

6. Abdelrazaq, A. Validating the Structural Behavior and Response of Burj Khalifa / A. Abdelrazaq // International Journal of High-Rise Buildings. - 2012. - Vol. 1. - № 1. - P. 37-51.

7. Belostotsky, A. Adaptive finite element models coupled with structural health monitoring systems for unique buildings / A.M. Belostotsky, P.A. Akimov // Procedia Engineering. - 2016. - Vol. 153/ - P. 8-15.

8. Strength and stability analysis of load-bearing structures of Evolution tower with allowance for actual positions of reinforced concrete structural memembers / A.M. Belostotsky, P.A. Akimov, T.B. Kaytukov [et al.] // Procedia Engineering. 2016. - Vol. 153. - P. 95-102.

9. Adaptive finite-element models in structural health monitoring systems / A.M. Belostotsky, P.A. Akimov, 0.A. Negrozov [etal.] // Инженерно-строительный журнал. - 2018. - № 2 (78). - C. 169-178.

10. Brownjohn, J.M.W. Structural health monitoring of civil infrastructure / J.M.W. Brownjohn // Philosophical Transactions of the Royal Society A. - 2007. - № 1851 (365). - P. 589-622.

11. Chang, P.C. Review paper: Health monitoring of civil infrastructure / P.C. Chang, A. Flatau, S.C. Liu // Structural Health Monitoring. - 2003. - № 2 (3). - P. 257-267.

12. Nonlinear finite element model updating for damage identification of civil structures using batch Bayesian estimation / H. Ebrahimian, R. Astroza, J.P. Conte, R.A. Callafon De // Mechanical Systems and Signal Processing. - 2017. - № 84 (B). - P. 194-222.

13. Friswell, M.I. Experimental test data: Parameterization and regularization / M.I. Friswell, J.E. Mottershead, H. Ahmadian // Transactions of the Royal Society of London, Series A, Special Issue on Experimental Modal Analysis. - 2001. - № 359 (1778). - P. 169-186.

14. Mathematical and computer modelling as the basis of structural health monitoring / T.B. Kaytukov, P.A. Akimov, V.N. Sidorov, A.M. Belostosky // IOP Conference Series: Materials Science and Engineering. - 2018. - Vol. 456. - 012072.

15. Kefal, A. An enhanced inverse finite element method for displacement and stress monitoring of multilayered composite and sandwich structures / A. Kefal, A. Tessler, E. Oterkus // Composite Structures. - 2017. - № 179. - P. 514-540.
16. Recent R\&D activities on structural health monitoring in Korea / J.-T. Kim, S.-H. Sim, S. Cho [et al.] // Structural Monitoring and Maintenance. - 2016. - № 1 (3). - P. 91-114.

17. Mordini, A. The finite element model updating: A powerful tool for structural health monitoring / A. Mordini, K. Savov, H. Wenzel // Structural Engineering International. 2007. - № 4. - P. 352-358.

18. Novikov, P.I. Identifying Real Stiffness Properties of Structural Elements of Adapted Finite-Element Models of Buildings and Structures. Part 1: Problem Setting / P.I. Novikov // Applied Mechanics and Materials. - 2014. - № 670-671. -P. 732-735.

19. Preliminary design of structural health monitoring for high-rise buildings / H.-H. Ryu, J.-S. Kim, E.-G. Choi, S.-H. Lee // International Journal of High-Rise Buildings. - 2017/ - № 3 (6). -P. 279-284.

20. Computational model for supporting SHM systems design: Damage identification via numerical analyses / M. Sartorato, Medeiros De R., D. Vandepitte, V. Tita // Mechanical Systems and Signal Processing. - 2017. - № 84 (A). - P. 445-461.

21. Long-term structural performance monitoring system for the Shanghai Tower / Su J.Z., Xia Y., Chen L. [et al.] // Journal of Civil Structural Health Monitoring. - 2013. - Vol. 3, Is. 1. - P. 49-61.

22. Suhaimi, S.A. Evolution of structural health monitoring / S.A. Suhaimi, S.N. Azemi, S.P. Jack// Journal of Built Environment, Technology and Engineering. - 2016. - № 1. - P. 76-80.

23. Deformation monitoring of super-tall structure using real-time strain data / Y. Xia, P. Zhang, Y. Ni, H. Zhu // Engineering Structures. - 2014. - Vol. 67. - P. 29-38.

24. Travush, V.I. Contemporary Digital Technologies in Construction. Part 1: About Mathematical (Numerical) Modelling / V.I. Travush, A.M. Belostotsky, P.A. Akimov//IOP Conference Series: Materials Science and Engineering. - 2018. - Vol. 456. - 012029.

25. Travush, V.I. Contemporary Digital Technologies in Construction. Part 2: About Experimental \& Field Studies, Material Sciences, Construction Operations, BIM and "Smart" City / V.I. Travush, A.M. Belostotsky, P.A. Akimov // IOP Conference Series: Materials Science and Engineering. - 2018. - Vol. 456. - 012029.

26. Ursos, M.E. A finite element based method for estimating natural frequencies of locally damaged homogeneous beams / M.E. Ursos, E.A. Tingatinga, R.E. Longalong // Procedia Engineering. - 2017. - № 199. - P. 404-410.

27. Structural health monitoring of Shangai tower considering time depending effect / Q. Zhang, B. Yang, T. Liu [et al.] // International journal of high-rise buildings. - 2015. - Vol. 4. - № 1. - P. 39-44.

28. A multiscale finite element model validation method of composite cablestayed bridge based on Probability Box theory / R. Zhong, Z. Zong, J. Niu [et al.] // Journal of Sound and Vibration. - 2016. -№ 370. - P. 111-131.

29. Zhou, H.Z. Recent advances in research on damage diagnosis for civil engineering structures / H.Z. Zhou // China Civil Engineering Journal. - 2003. - № 36 (5). - P. 105-110. 


\section{References}

1. Belostotsky A.M., Akimov P.A. Nauchno-issledovatelskiy tsentr StaDy0. 25 let na fronte chislennogo modelirovaniya [25-th Anniversary of scientific research centre StaDy0]. International Journal for Computational Civil and Structural Engineering, 2016, Vol. 12, Iss.1, pp. 9-34 (in Russian).

2. Emanov A.F. V Vosstanovlenie kogerentnykh sostavlyayushchikh volnovykh polei v seismike [Recovery of coherent components of wave fields in seismic]. The dissertation for the degree of Doctor of Technical Sciences in the specialty 25.00.10 "Geophysics, geophysical methods of searching for minerals". Novosibirsk, Altai-Sayan branch of the Geophysical Service, Siberian Branch of the Russian Academy of Sciences, 2004, 279 p. (in Russ.)

3. Emanov A.F., Krasnikov A.A. Primenenie metoda stoyachikh voln dlya issledovanii seismoizolirovannykh zdanii [The use of standing waves method in study of seismically isolated buildings]. Voprosy inzhenernoi seismologii [Problems of Engineering Seismology], 2015, no. 4 (42), pp. 37-64. (in Russ.)

4. Kalichava D.K. Adaptivnye dinamicheskie konechnoelementnye modeli $v$ osnove monitoringa nesushchikh konstruktsii vysotnykh zdanii [Adaptive dynamic finite element models based on monitoring of load-bearing structures of high-rise buildings]. The dissertation for the degree of candidate of technical sciences in the specialty 05.13.18 "Mathematical modeling, numerical methods and program complexes". Moscow, Moscow State University of Civil Engineering, 2012, 149 p. (in Russ.)

5. Krasnikov A.A., Yemanov A.F., Bakh A.A. Otsenka polnoty konechno-elementnykh modelei inzhenernykh sooruzhenii po eksperimental'nym dan-nym metoda stoyachikh voln [Finite element model of engineering structures completeness assessment by standing waves method experimental data]. Interekspo Geo-Sibir, 2017, no. 4(2), pp. 179-184. (In Russ.).

6. Abdelrazaq A. Validating the Structural Behavior and Response of Burj Khalifa. International Journal of High-Rise Buildings, 2012, Vol. 1, no. 1, pp. 37-51.

7. Belostotsky A.M., Akimov P.A. Adaptive finite element models coupled with structural health monitoring systems for unique buildings. Procedia Engineering, 2016, Vol. 153, pp. 8-15.

8. Belostotsky A.M., Akimov P.A., Kaytukov T.B., Petryashev N.0., Petryashev S.0., Negrozov 0.A. Strength and stability analysis of load-bearing structures of Evolution tower with allowance for actual positions of reinforced concrete structural memembers. Procedia Engineering, 2016, Vol. 153, pp. 95-102.

9. Belostotsky A.M., Akimov P.A., Negrozov 0.A. Petryashev N.O., Petryashev S.0., Sherbina S.V., Kalichava D.K., Kaytukov T.B. Adaptive finite-element models in structural health monitoring systems. Inzhenerno-stroitel'nyi zhurnal [Magazine of Civil Engineering], 2018, no. 2 (78), pp. 169-178.

10. Brownjohn J.M.W. Structural health monitoring of civil infrastructure. Philosophical Transactions of the Royal Society A, 2007, no. 1851 (365), pp. 589-622.
11. Chang P.C., Flatau A., Liu S.C. Review paper: Health monitoring of civil infrastructure. Structural Health Monitoring, 2003, no. 2 (3), pp. 257-267.

12. Ebrahimian H., Astroza R., Conte J.P. De Callafon R.A. Nonlinear finite element model updating for damage identification of civil structures using batch Bayesian estimation. Mechanical Systems and Signal Processing, 2017, no. 84 (B), pp. 194-222.

13. Friswell M.I., Mottershead J.E., Ahmadian H. Experimental test data: Parameterization and regularization. Transactions of the Royal Society of London, Series A, Special Issue on Experimental Modal Analysis, 2001, no. 359 (1778), pp. 169-186.

14. Kaytukov T.B., Akimov P.A., Sidorov V.N., Belostosky A.M. Mathematical and computer modelling as the basis of structural health monitoring. IOP Conference Series: Materials Science and Engineering, 2018, Vol. 456, 012072.

15. Kefal A., Tessler A., Oterkus E. An enhanced inverse finite element method for displacement and stress monitoring of multilayered composite and sandwich structures. Composite Structures, 2017, no. 179, pp. 514-540.

16. Kim J.-T., Sim S.-H., Cho S., Yun C.-B., Min J. Recent R\&D activities on structural health monitoring in Korea. Structural Monitoring and Maintenance, 2016, no. 1(3), pp. 91-114.

17. Mordini A., Savov K., Wenzel H. The finite element model updating: A powerful tool for structural health monitoring. Structural Engineering International, 2007, no. 4, pp. 352-358.

18. Novikov P.I. Identifying Real Stiffness Properties of Structural Elements of Adapted Finite-Element Models of Buildings and Structures. Part 1: Problem Setting. Applied Mechanics and Materials, 2014, no. 670-671, pp. 732-735.

19. Ryu H.-H., Kim J.-S., Choi E.-G., Lee S.-H. Preliminary design of structural health monitoring for high-rise buildings. International Journal of High-Rise Buildings, 2017, no. 3(6), pp. 279-284.

20. Sartorato M., De Medeiros R., Vandepitte D., Tita V. Computational model for supporting SHM systems design: Damage identification via numerical analyses. Mechanical Systems and Signal Processing, 2017, no. 84 (A), pp. 445-461.

21. Su J.Z., Xia Y., Chen L., Zhao X., Zhang Q.L., Xu Y.L., Ding J.M., Xiong H.B., Ma R.J., Lv X.L., Chen A.R. Long-term structural performance monitoring system for the Shanghai Tower. Journal of Civil Structural Health Monitoring, 2013, Vol. 3, Iss. 1, pp. 49-61.

22. Suhaimi S.A., Azemi S.N., Jack S.P. Evolution of structural health monitoring. Journal of Built Environment, Technology and Engineering, 2016, no. 1, pp. 76-80.

23. Xia Y., Zhang P., Ni Y., Zhu H. Deformation monitoring of super-tall structure using real-time strain data. Engineering Structures, 2014, Vol. 67, pp. 29-38.

24. Travush V.I., Belostotsky A.M., Akimov P.A. Contemporary Digital Technologies in Construction Part 1: About Mathematical (Numerical) Modelling. IOP Conference Series: Materials Science and Engineering, 2018, Vol. 456, 012029. 
25. Travush V.I., Belostotsky A.M., Akimov P.A. Contemporary Digital Technologies in Construction Part 2: About Experimental \& Field Studies, Material Sciences, Construction Operations, BIM and "Smart" City. IOP Conference Series: Materials Science and Engineering, 2018, Vol. 456, 012029.

26. Ursos M.E., Tingatinga E.A., Longalong R.E. A finite element based method for estimating natural frequencies of locally damaged homogeneous beams. Procedia Engineering, 2017, no. 199, pp. 404-410.

27. Zhang Q., Yang B., Liu T., Li H., Lv J. Structural health monitoring of Shangai tower considering time depending effect.
International journal of high-rise buildings, 2015, Vol. 4, no. 1, pp. 39-44.

28. Zhong R., Zong Z., Niu J., Liu Q., Zheng P. A multiscale finite element model validation method of composite cablestayed bridge based on Probability Box theory. Journal of Sound and Vibration, 2016, no. 370, pp. 111-131.

29. Zhou H.Z. Recent advances in research on damage diagnosis for civil engineering structures. China Civil Engineering Journal, 2003, no. 36 (5), pp. 105-110.

Белостоцкий Александр Михайлович (Москва). Доктор технических наук, профессор, член-корреспондент РААСН. Генеральный директор Научно-исследовательского центра «СтаДи0» (125124, Москва, ул. 3-я Ямского Поля, д.18, оф. 810. НИЦ «СтаДи0»). Эл.почта: amb@stadyo.ru.

Акимов Павел Алексеевич (Москва). Доктор технических наук, профессор, академик РААСН. Временно исполняющий обязанности ректора ФГБОУ В0 «Национальный исследовательский Московский государственный строительный университет» (129337, Москва, Ярославское шоссе, 26. НИУ МГСУ). Эл. почта: pavel.akimov@gmail.com.

Кайтуков Таймураз Батразович (Москва). Кандидат технических наук, доцент, советник РААСН. Проректор ФГБОУ В0 «Национальный исследовательский Московский государственный строительный университет» (129337, Москва, Ярославское шоссе, 26. НИУ МГСУ). Эл. почта: tkaytukov@gmail.com.

Петряшев Николай Олегович (Москва). Инженер Центрального научно-исследовательского института строительных конструкций (ЦНИИСК) им. В.А. Кучеренко АО «Научно-исследовательский центр “Строительство”» (109428, Москва, ул. 2-я Институтская, 6. ЦНИИСК им. В.А. Кучеренко). Эл. почта: petryashev.n.o@gmail.com.

Петряшев Сергей Олегович (Москва). Инженер Центрального научно-исследовательского института строительных конструкций (ЦНИИСК) им. В.А. Кучеренко АО «Научно-исследовательский центр “Строительство”» (109428, Москва, ул. 2-я Институтская, 6. ЦНИИСК им. В.А. Кучеренко). Эл. почта: petsero@mail.ru.

Belostotsky, Alexander M. (Moscow). Doctor of Technical Sciences, Professor, Corresponding Member of RAACS. General Director of the Research Center "StaDi0" (18 3-ya Yamskogo Polya St., office 810, Moscow, 125124. SRC StaDy0). E-mail:amb@stadyo.ru.

Akimov, Pavel A. (Moscow). Doctor of Technical Sciences, Professor, Full Member of the RAACS. Acting Rector of the National Research Moscow State University of Civil Engineering (26 Yaroslavskoe Shosse, Moscow, 129337, NRU MGSU). E-mail: pavel.akimov@gmail.com.

Kaytukov, Taymuraz B. (Moscow). Candidate of Technical Sciences, Associate Professor, Advisor of the RAACS. Vice-Rector of the National Research Moscow State University of Civil Engineering (26 Yaroslavskoe Shosse, Moscow, 129337, NRU MGSU). E-mail: tkaytukov@gmail.com.

Petryashev, Nikolay 0. (Moscow). Engineer of the Research Institute of Building Constructions (TSNIISK) named after V.A. Koucherenko A0 "Scientific-Research Center "Construction" (6 2-ya Institutskaya St., Moscow, 109428. TSNIISK). Email: petryashev.n.o@gmail.com.

Petryashev, Sergey 0. (Moscow). Engineer of the Research Institute of Building Constructions (TSNIISK) named after V.A. Koucherenko AO "Scientific-Research Center "Construction" (6 2-ya Institutskaya St., Moscow, 109428. TSNIISK). E-mail: petsero@mail.ru. 